\title{
The Study on Measurement Error of Strip-line Resonator Method Hao $\mathrm{Li}^{1,}$, , Sen Wang ${ }^{2, b}$ and Shuo Cui ${ }^{3, \mathrm{c}}$ \\ ${ }^{1}$ NO.1 Nan Da Hong Men Road, Fengtai District, Beijing, China \\ ${ }^{2}$ NO.1 Nan Da Hong Men Road, Fengtai District, Beijing, China \\ ${ }^{3}$ No.2006, Xiyuan Ave, West Hi-Tech Zone, Chengdu,.China \\ a952077096@qq.com, b15908109077@163.com, c cshuofred@foxmail.com
}

Keywords: Strip-line resonator, Complex permittivity of material, Surface resistance.

Abstract. High-frequency PCB is widely used in the microwave devices, such as RF circuits and patch antennas. The performance of the PCB is mainly determined by the parameter of complex permittivity. There are many methods to measure the complex permittivity of the PCB. Among these methods, strip-line resonator method is the best test method because of its high accuracy and simple fixture. In order to improve the test accuracy of this method further, this paper consider the influence of the coupling gap, central conductor width, high order modes and surface resistivity by simulated and experimental analysis. The results show that the surface resistivity of the ground has a great impact on the quality factor of the strip-line resonator. So we use the parallel-plate dielectric resonator method to measure the surface resistivity under variable temperature, and then present a new amendments method.

\section{Introduction}

Based on the basic theory of strip-line resonators[1], the resonance frequency and quality factor of strip-line resonator is the most important data to calculate the complex permittivity of the material, and each parameter of resonator structure have a great influence on the two data.Therefore, it is necessary to have a thorough understanding of the influence on the resonant frequency and quality factor of resonator when these parameters change.

\section{Error Analysis}

The Influence of the Coupling Gap. In order to have a complete and intuitive understanding of the effect of gap size on the frequency response of a strip-line resonator, we need the simulation of different size of the coupling interval strip-line resonator. By observing the simulation results, we can control the coupling gap size to fix strip-line resonator.

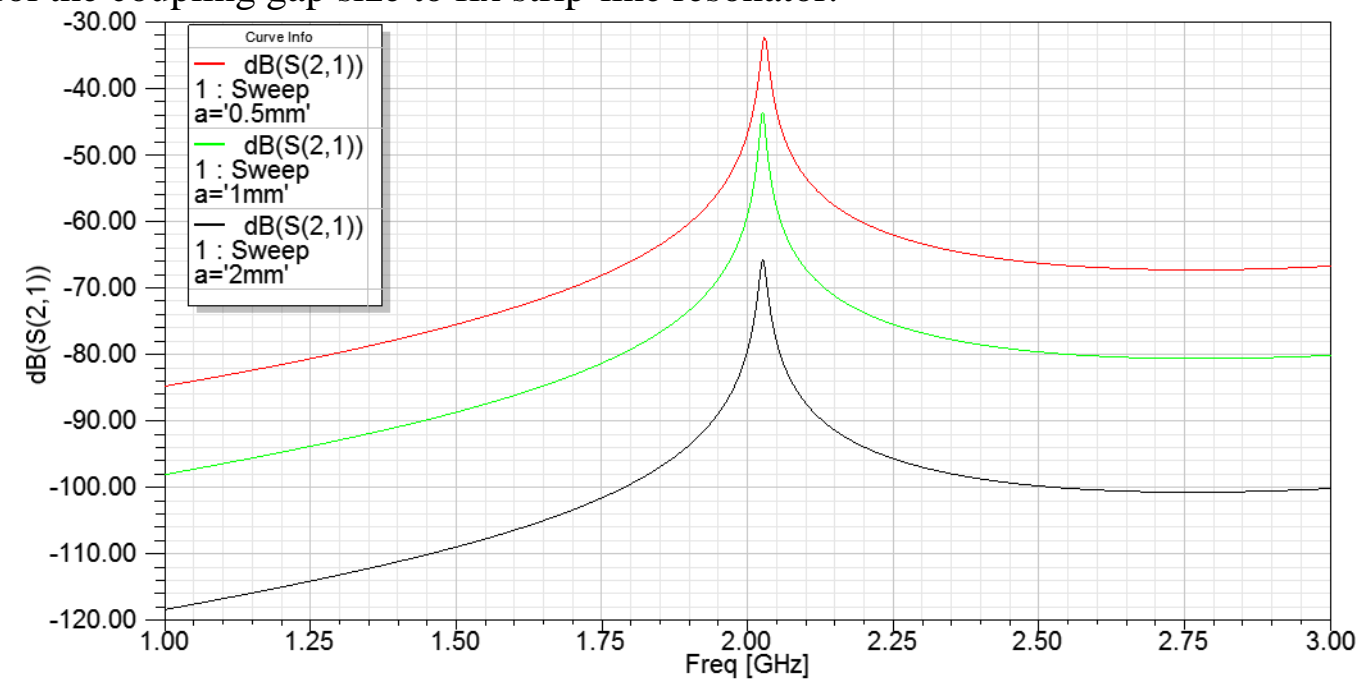

Fig. $1 \mathrm{~S} 21$ of resonator on $2 \mathrm{GHz}$. 


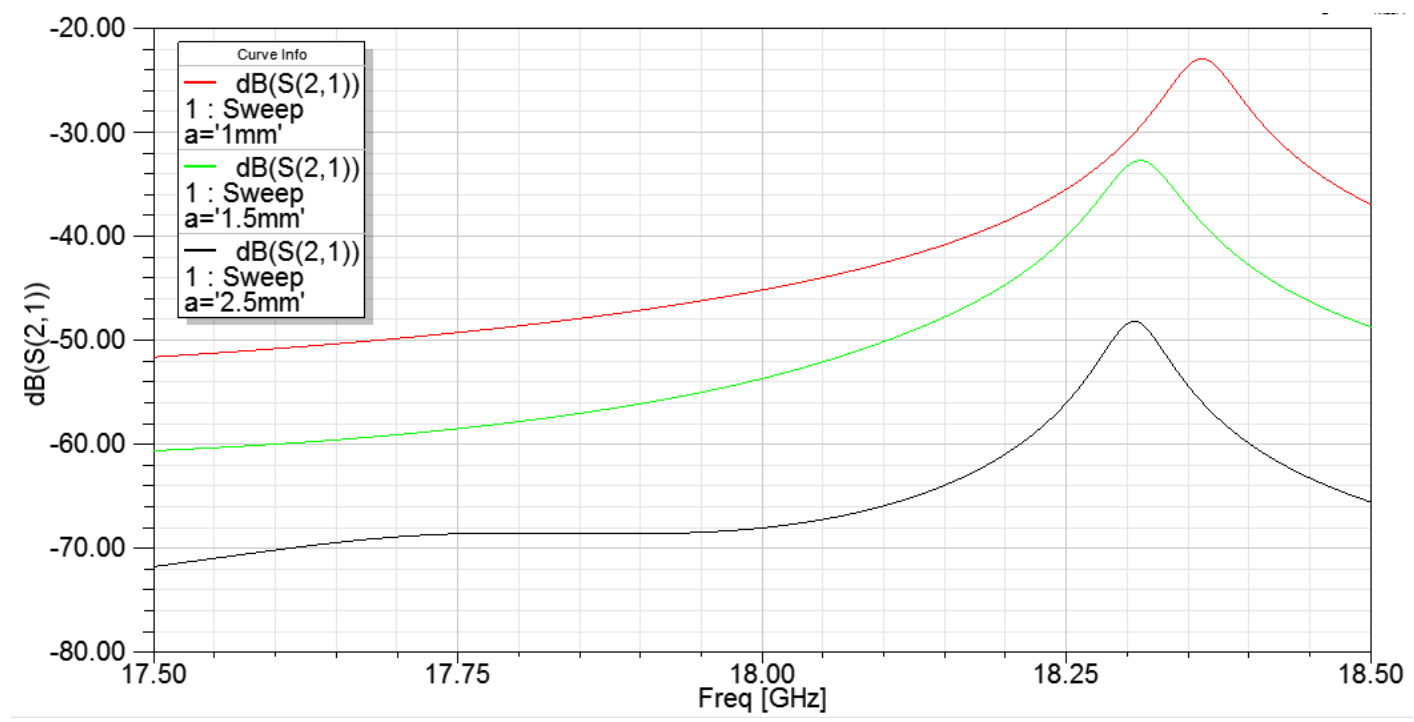

Fig. 2 S21 of resonator on $18 \mathrm{GHz}$.

Based on the simulation and measured results, we can get the following conclusion:

1. The smaller coupling gap can obtain more obvious resonance peak, but also cause decline of the quality factor, seriously affect the test accuracy and test frequency range.

2. At high frequencies, the influence of the coupling gap will become more complex (associated with the gap size). This phenomenon may cause singularity problem in testing of dielectric loss value.

The Influence of Central Conduction Band Width. To observe the effect of center conduction band's width on the response of strip-line resonator, taken Rogers6010 as example, testing resonance frequency when center conduction band`s width is $1 \mathrm{~mm}, 2 \mathrm{~mm}, 3 \mathrm{~mm}$ and the frequency is about $10 \mathrm{GHz}$.

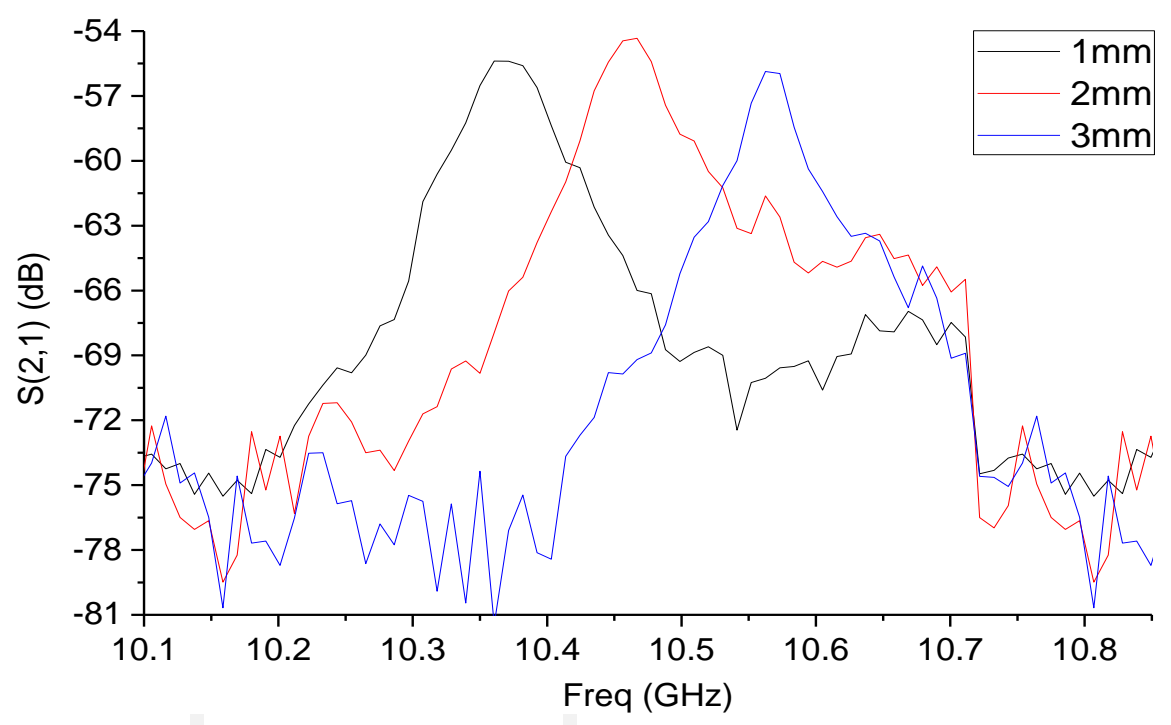

Fig. 3 S21 of resonator with different width of conduction band.

By observing the test result, the Fig. 3 shows that as the width increase, the resonance peak frequency increases. Therefore the corresponding relative dielectric constant was smaller.

The Influence of High Order Mode. Taken Rogers6010 as example,through the test we can observe the effects of high order mode of resonance peak. 


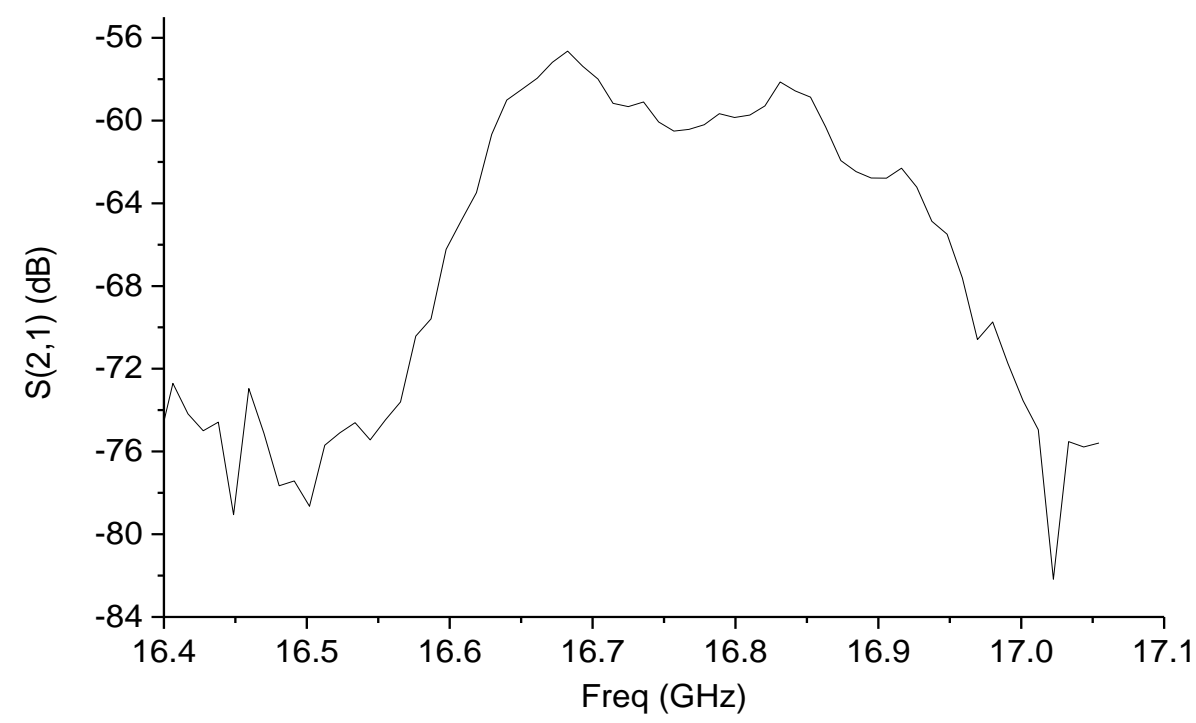

Fig. 4 S21 of resonator on $16.7 \mathrm{GHz}$.

We can get the following conclusion through the test result:

1. Strip-line resonator will produce a high order mode at high frequencies.

2. High order mode will have an influence on the quality factor, seriously affect the test accuracy and test frequency range.

The Influence of Conductor Surface Resistance. When the temperature of the test environment changes, the surface resistivity [2] is bound to change, if we ignore this change, the material loss will not reliable. The measurement of conductor surface resistivity are described in the next section, this section will use the HFSS simulation different surface resistivity for strip-line resonator quality factor at $10 \mathrm{GHz}$.

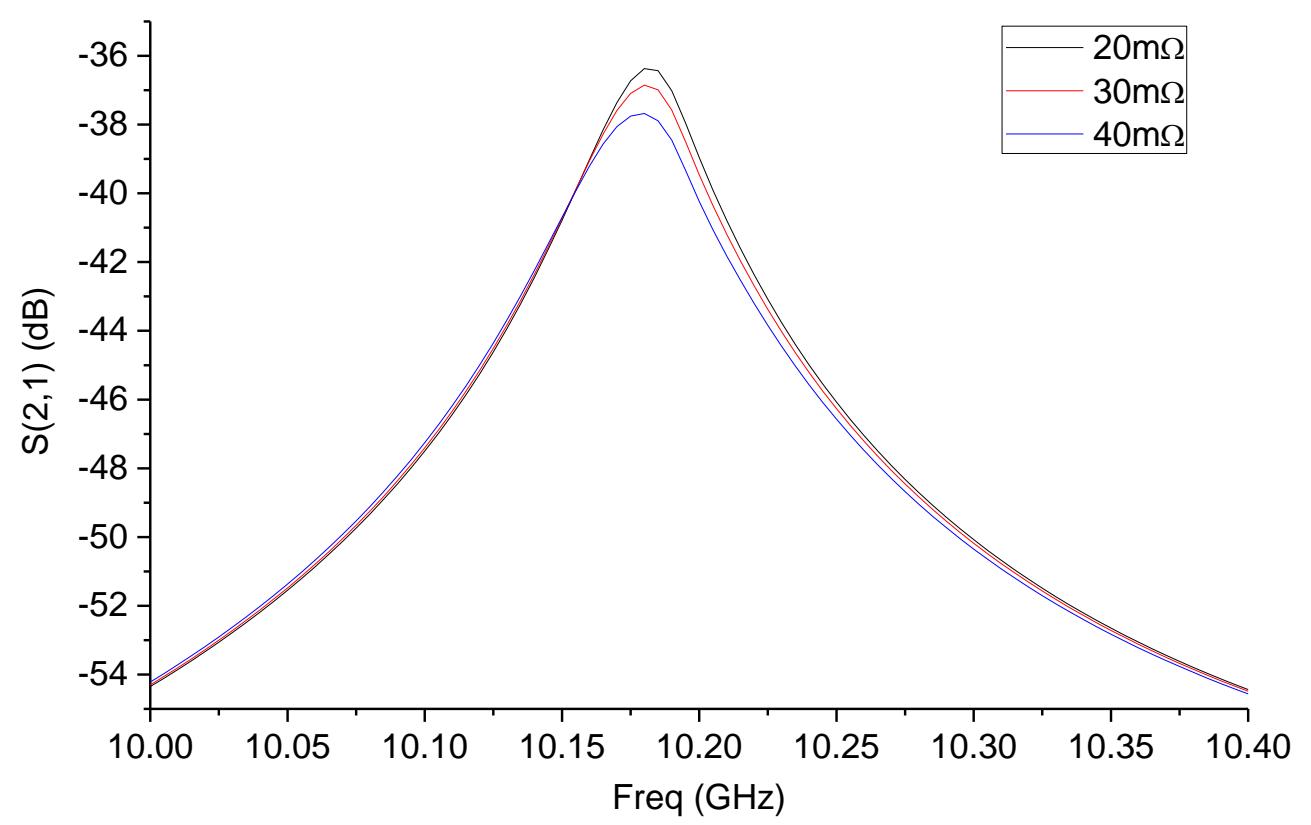

Fig. 5 S21 of resonator with different surface resistivity.

As shown in Fig. 5, the surface resistivity changes will significantly affect the quality factor of resonator; therefore the accurate measurement of the surface conductor resistivity at variable temperature can improve the test precision of the dielectric loss.

\section{Surface Resistance Measurement}

Parallel plate dielectric resonator method, also called Courtney method [4], it is made by a cylindrical dielectric resonator, whose both ends are conductor plate or superconducting plate 
structure. The structure is the simplest and the most suitable to the measurement of microwave surface resistance.Then we will introduce the theory of parallel plate dielectric resonator method.

The unloaded quality factor of the resonatorcan $Q_{u}$ be expressed as:

$$
\frac{1}{Q_{u}}=\frac{1}{Q_{c}}+\frac{1}{Q_{d}}+\frac{1}{Q_{r}}
$$

where, $Q_{u}$ indicates that the quality factor of the conductor, $Q_{d}$ represents the quality factor of the medium, $Q_{r}$ represents the radiation quality factor, and each quality factor can be expressed as:

$$
Q_{i}=\frac{2 \pi f_{r} W_{0}}{P_{i}}
$$

Where $W_{0}$ represents the total energy storage of the system, $f_{d}$ represents the resonant frequency, then dielectric quality factor can be expressed as:

$$
\frac{1}{Q_{d}}=\frac{P_{d}}{2 \pi f_{r} W_{0}}=\frac{W_{E 1}}{W_{0}} \tan \delta=\frac{W_{E 1}}{W_{E 1}+W_{E 2}} \tan \delta=\frac{\tan \delta}{1+w}
$$

$W_{E 1}$ and $W_{E 2}$ respectively represent internal and external energy storage of the cylindrical dielectric resonators. The power loss of the conductor plate can be approximately expressed as:

$$
P_{c}=\frac{1}{2} \int_{S} R_{S}\left|\overrightarrow{J_{S}}\right|^{2} d S=\frac{1}{2} R_{S} \int_{S}|\vec{H}|^{2} d S
$$

$R_{s}$ represents the surface resistivity of the conductor, $J_{s}$ and $\mathrm{H}$ respectively represent the ideal conductor surface current and magnetic field. This approximation is also applicable to a good conductor in the microwave frequency. We assume that $R_{s}$ is a variation when the temperature changes.

The expression $R_{s}$ can be obtained by the formula (4).

$$
R_{S}=\frac{2 \pi f_{r} W_{0}}{\int_{S}|\vec{H}|^{2} d S}\left(\frac{1}{Q_{u}}-\frac{\tan \delta}{1+w}-\frac{\mathrm{P}_{\mathrm{r}}}{2 \pi f_{r} W_{0}}\right)
$$

The value of $W_{0}, P_{r}$ and $\mathrm{W}$ is determined by the electromagnetic field distribution of $T E_{011}$ mode $[3,4]$, and the exact expression of the electromagnetic field components can be derived from an infinite conductor plate. In the case of infinite size plate $\left(P_{r}=0\right)$, the exact expression of the electromagnetic field components and the total electric field energy storage (5) can be expressed as:

$$
R_{S}=\frac{240 \pi^{2} \varepsilon_{r}}{1+\varepsilon_{r} w}\left(\frac{L}{\lambda}\right)^{3}\left(\frac{1+w}{Q_{u}}-\tan \delta\right)
$$

The loss tangent value and relative dielectric constant are known before measurement, and resonant frequency $f_{r}$ and unload quality factor are obtained by measurement.

The $R_{s}$ in Formula (6) is derived in the case of infinite conductor plate. We need to make the appropriate correction for the actual limited conductor plate. By using the approximate formula in reference [5]:

$$
R_{s}=\frac{240 \pi^{2}}{Q_{c}}\left(\frac{L f_{0}}{c}\right) \frac{1+R}{1+\varepsilon_{r} R}
$$

We use the reference data previously measured of the sapphire standard sample as a reference, and then we tested the surface resistance of the copper foil of strip-line resonator samples on some discrete temperature point. The resonance frequency and $\mathrm{Q}$ value measured under different temperature are shown in Table 1. 
Table 1. Test result under different temperature.

\begin{tabular}{|c|c|c|c|}
\hline Test temperature & $\begin{array}{c}\text { Resonant } \\
\text { frequency }(\mathrm{GHz})\end{array}$ & $\begin{array}{c}\mathrm{Q} \\
\text { (quality factor) }\end{array}$ & $\begin{array}{c}\text { Rs } \\
\text { (surface resistance) }\end{array}$ \\
\hline$-40^{\circ} \mathrm{C}$ & 11.293 & 6994 & $24.69 \mathrm{~m} \Omega$ \\
\hline$-20^{\circ} \mathrm{C}$ & 11.280 & 6533 & $25.94 \mathrm{~m} \Omega$ \\
\hline $0^{\circ} \mathrm{C}$ & 11.269 & 6239 & $27.50 \mathrm{~m} \Omega$ \\
\hline $40^{\circ} \mathrm{C}$ & 11.261 & 5857 & $31.19 \mathrm{~m} \Omega$ \\
\hline $80^{\circ} \mathrm{C}$ & 11.248 & 5465 & $35.03 \mathrm{~m} \Omega$ \\
\hline
\end{tabular}

The final measured surface resistivity of the metal will be substituted into the algorithm of strip-line resonator to obtain the corrected dielectric loss parameters.

Table 2. Rogers R/T $600640^{\circ} \mathrm{C}$ test results.

\begin{tabular}{|c|c|c|c|}
\hline $\begin{array}{c}\text { Resonant frequency } \\
(\mathrm{MHz})\end{array}$ & permittivity & Loss tangent & $\begin{array}{c}\text { Loss tangent } \\
\text { (modification value) }\end{array}$ \\
\hline 8408.466 & 6.23 & 0.0016 & 0.0015 \\
\hline 9616.71 & 6.22 & 0.0021 & 0.002 \\
\hline 10805.95 & 6.23 & 0.0024 & 0.0023 \\
\hline 12006.37 & 6.23 & 0.0023 & 0.0022 \\
\hline
\end{tabular}

Compared the test data of Rogers RT/Duroid 6006 samples under 40 degrees Celsius with reference data provided by Rogers, we find out that the permittivity and loss tangent showed good consistency.

\section{Conclusions}

In this paper, we first introduce the effect of coupling gap, the width of centric strip-line conductor and the surface conductor resistivity on the frequency response strip-line resonator.After that we focused on the dielectric resonator method to measurement of the surface resistivity of conductor, and then we get the method of correction of surface resistivity through the theoretical analysis. Finally, we tested the surface resistivity and substituted Rs to the algorithm again.Therefore we can obtain the modified dielectric loss parameters. Based on the findings the following conclusions can be derived as:

1. The smaller coupling gap can obtain more obvious resonance peak, but also cause decline of the quality factor, seriously affect the test accuracy and test frequency range.

2. As the central conduction band width increase, the resonance peak frequency increases. Therefore the corresponding relative dielectric constant was smaller.

3. Strip-line resonator will produce a high order mode at high frequencies, and high order mode will have an influence on the quality factor, seriously affect the test accuracy and test frequency range.

4. The surface resistivity changes will significantly affect the quality factor of resonator.

Through the above analysis, we can say that it is of great significance to improve the test accuracy of strip-line resonator method.

\section{References}

[1] X.Q.Wang, The Measurement of Complex Permittivity of Dielectric Materials Under Low temperature Condition, University of Electronic Science and Technology of China, (2015).

[2] A. Portis, K. Blazey, K. Müller, et al. Microwave Magneto-Surface Impedance of High-Tc Super conductors, EPL (Europhysics Letters), 5(5) (1988) 467.

[3] J. Krupka, K. Derzakowski, et al. A Dielectric Resonator for Measurements of Complex Permittivity of Low Loss Dielectric Materials as a Function of Temperature, Measur. Sci. Technol. 9(10) (1998) 1751. 
[4] W. E. Courtney, Analysis and Evaluation of a Method of Measuring the Complex Permittivity and Permeability Microwave Insulators, Microwave Theory and Techniques, IEEE T. 18(8) (1970) 476-485.

[5] A. Mourachkine, A. R. Barel, Microwave Measurement of Surface Resistance by the Parallel-Plate Dielectric Resonator Method, Microwave Theory and Techniques, IEEE T. 43(3) (1995) 544-551. 